\title{
Ubiquitous Wireless Power Transfer for Multiple Mobile Devices
}

\author{
Alexander Decker de Sousa ${ }^{1}$, Luiz Filipe Menezes Vieira ${ }^{1}$, \\ Marcos Augusto Menezes Vieira ${ }^{1}$ \\ ${ }^{1}$ Computer Science Department - Universidade Federal de Minas Gerais (UFMG) \\ Belo Horizonte - MG - Brazil \\ \{alexanderdecker, lfvieira,mmieira\}@dcc.ufmg.br
}

\begin{abstract}
We propose two new computational problems associated with the charging of mobile devices using wireless power transfer via magnetic induction. Algorithms for these problems may enable ubiquitous charging, meaning the user is no longer required to be aware of the devices charging processes. We prove both problems as being NP-Hard and propose three dynamicprogramming algorithms to solve them in linear time regarding the size of the time-horizon. We also propose three greedy algorithms for the problems. Experiments indicate that the best dynamic-programming algorithm among those proposed reaches between $89 \%$ and $97 \%$ of effectiveness, while the best greedy reaches between $74 \%$ and $92 \%$, depending on the considered scenario.
\end{abstract}

\section{Introduction}

The global market of wireless charging devices was valued at $\$ 6,514.2$ million in 2018 and is projected to reach \$ 49,304.1 million by 2027 [Patil 2020]. Most of these devices use Inductive Power Transfer (IPT) protocols as physical layer. Although historically short-ranged, those protocols recently became more comprehensive by exchanging information between power transmitters and receivers using wireless links [Shi et al. 2015]. However, most wireless charging solutions in the literature do not address the charging process in their modeling, often focusing on wireless power transfer (WPT) [Jadidian and Katabi 2014]. Indeed, maximizing the power transfer is equivalent to minimize the charging time and maximize the life-time of the devices if the system features a single power receiver or a single power transmitter. However, the problems became quite different for multiple transmitters and receivers.

The two problems proposed by this work start filling a gap between the approach employed by the aforementioned works and the charging processes of the powered devices. The Minimum-Time MIMO Charging Problem applies to cases where a Multiple-Input-Multiple-Output (MIMO) IPT transmitting station must charge a set of nodes as soon as possible. Use cases include the automated charging of wireless nodes [Xie et al. 2012] and the power distribution among unmanned aerial vehicles (UAVs) in Flying Ad hoc Networks (FANETS) [Bekmezci et al. 2013]. The automated charging of wireless nodes is often implemented using a wireless transmitting vehicle that runs within the network area and meanwhile provides the power for charging the nodes. Thus, charging the nodes in a certain sub-area as fast as possible is fundamental for the vehicle to attend other sub-areas without disconnections due to energy issues. For the power distribution among FANETs, in turn, the nodes must approach each other for 
the power transmission to occur, so the main purposes of the network may be temporally prejudiced and, therefore, it is required for the charging process to be agile. Besides that, UAVs face landing inaccuracies that might lead to conditions of poor coupling with the transmitter. Thus, a MIMO setup can improve tolerance to misalignment and distance.

The No-Starvation MIMO Charging Problem, in turn, is related to ubiquitous wireless power charging. The term refers to the paradigm where users of mobile devices remain oblivious to battery charging due to the ubiquity of transmitters and the management of the charges. In addition to usability improvements, the paradigm offers mobility gains in Wireless and Ubiquitous Networks, as devices can physically disconnect from power sources without losing ubiquity. The Ubiquitous WPT has already some glimpses in literature. Huang et al. [Huang et al. 2012] describes a simple power transmitter based on flexible sheets and solar panels, which is simple enough to allow extensive implantation. Assuming that mobile receivers are always close enough to the populations of transmitting devices, the priority is no longer to minimize charging time. Indeed, if the devices are not expected to stay out-of-reach for long periods, the transmitting controller might just manage the available resources in a way to fulfill the energy requirements of the receivers around. However, if a particular device expects to move away from the transmitter population soon, it can simply send a leaving-notification to the controller so that it seeks to ensure a sufficient charge while the device is still reachable.

The main contributions of this dissertation are: (i) the definition two new computational problems involving the process of charging multiple devices using MIMO inductive power transfer systems, (ii) the proof that both proposed problems belong to NPHard complexity class, (iii) the proof that the charging approach used by the previous work is sub-optimal regarding network lifetime and charging time, (iv) the proposition of a dynamic-programming method to solve both problems in linear time regarding the duration of the considered time horizon, (v) three algorithms for solving each proposed problem using the dynamic-programming method, (vi) three other algorithms for each proposed problem using greedy approaches, and (vii) an algorithm for generating random instances of the proposed problems which have guaranteed solution.

\section{Related Work}

Runtime Optimization of WPT. Systems with more than one IPT transmitting device may follow the beam-forming approach, which roughly enables the transmitting range and misalignment-tolerance to be improved [Jadidian and Katabi 2014, Shi et al. 2015]. Unlike the previous work, ours addresses the optimization of the whole charging process, instead of the simple power transfer.

Charging Optimization. As far as we know, all works which address the optimization of the wireless charging process instead of simply optimizing the power transference focus on scheduling of wireless nodes in wireless sensor networks. In short, these works address the optimization of the battery charges by selecting sub-networks to be prioritized by a single power-transmitting vehicle at each time-interval [Zhao et al. 2020, Lin et al. 2019]. Unlike these works, we consider a MIMO setup, which allows beamforming and enables the transmitting-voltages to be used as decision variables. Indeed, maximizing the transferred power with a single transmitter is a polynomial-time problem and might be solved by the exploration method described on page 40 of the dissertation. 
Wireless Energy Distribution. Some works focused on the distribution of energy across populations of power transceiver devices. Most works abstracted the WPT method and focused on optimizing the scheduling of the devices to be charged [Nikoletseas et al. 2017, Madhja et al. 2018]. As a wireless energy distribution technology, this work differs from its predecessors for (i) admitting charge limits within each battery can operate, (ii) considering bases dedicated to power transmission, and (iii) aiming, under ideal conditions, that users do not have to worry about the explicit charging of their devices and, therefore, the power sourcing of their devices be truly ubiquitous.

\section{Primer}

For a better understanding of the rest of this text, we provide some definitions. We consider a wireless charging setup as being a population of IPT transmitting devices spread across an environment together with a population of receiving devices. Both kinds of elements are illustrated by Figure 1. The transmitting devices have a centralized control which can determine the input voltage of each active circuit as long as the maximum power and the maximum current constraints are respected. Each receiving device is composed of a passive circuit which receives the power, a chargeable battery and a consumer device such as a cellphone or a electric vehicle. The charging vector of a given time-slot express the state of the system and consists of the instantaneous charge of every battery. Analogously, the input-voltage vector consists of the input-voltage of each active circuit at a given time-slot. Although Section 5.7 of the dissertation discuss the parameter acquisition, we often abstract sensing aspects and focus on the computational problems.
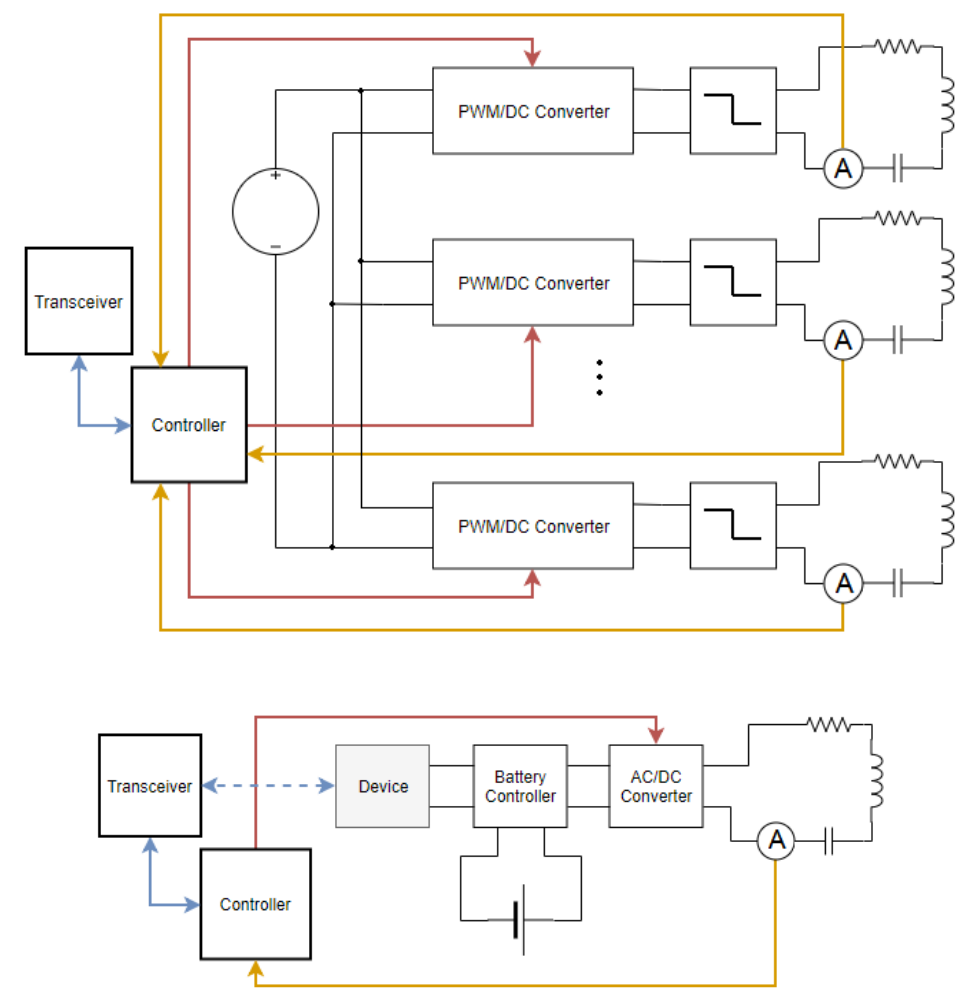

Figure 1. Scheme of the considered circuits used for transmitting and receiving power. 


\section{Theoretical Contributions}

This dissertation proposes two new computational problems regarding the prolonging of the battery autonomy of wireless devices using WPT. The No-Starvation MIMO Charging Problem consists of finding the input-voltage time-series of each transmitting device in order to provide all needed power to every receiving device within a given time-horizon. The input-voltage set of each time-slot of the horizon must be chosen in a way that the maximum power constraint is respected. Moreover, each electric current must respect the maximum allowed for each device and all devices batteries must end the charging process with more than a minimal charge. The Minimum-Time MIMO Charging Problem, in turn, consists of finding the input-voltage time-series which minimizes the charging-time. Analogously, the maximum power and maximum current constraints must be respected at each time-slot and all devices batteries must end with more than a minimal charge.

We demonstrate that the approach of the previous work, that is, always maximizing the immediately received power, is sub-optimal and may lead to very unfavorable scenarios, although the average case has good results. We prove that both proposed problems belong to the NP-Hard complexity class by providing polynomial-time reductions from the well known NP-Hard 0-1 Knapsack Problem.

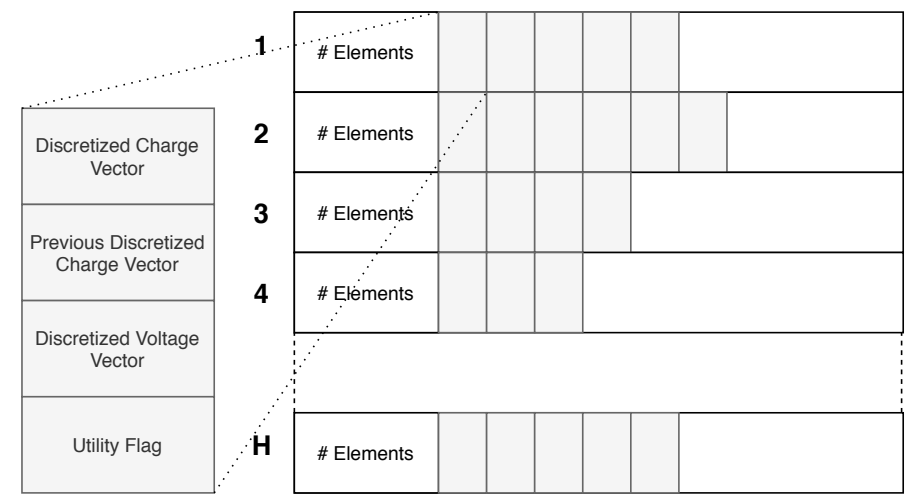

Figure 2. The simplified data structure used to store the charge-vector set of each time slot.

We propose a dynamic-programming method to solve both problems in lineartime regarding the number of time-slots in the time-horizon. Thereunto, we discretize the time and battery-charge vectors and define a hash-based memory structure which contains one set of charge-vectors for each time-slot. The employed data structure and the execution times of the main associated operations are better described in Figures 2 and 3. One charge-vector $q_{t}$ is considered to be "reachable" from a charge-vector $q_{t-1}$ from the previous time-slot if and only if there is at least one input-voltage vector which transitions $q_{t-1}$ to $q_{t}$ while respecting all constraints.

Thus, the method to find a solution for an instance of the No-Starvation MIMO Charging Problem is as illustrated by Figure 4. Starting from a single initial chargevector, we first populate the memory structure of the first time-slot with charge-vectors reachable from the initial one. Then, we populate the next time-slot using charge-vectors reachable from the previous set and so on. If there is at least a valid charge-vector in the last set, we walk the way back and build the solution. The method for an instance 


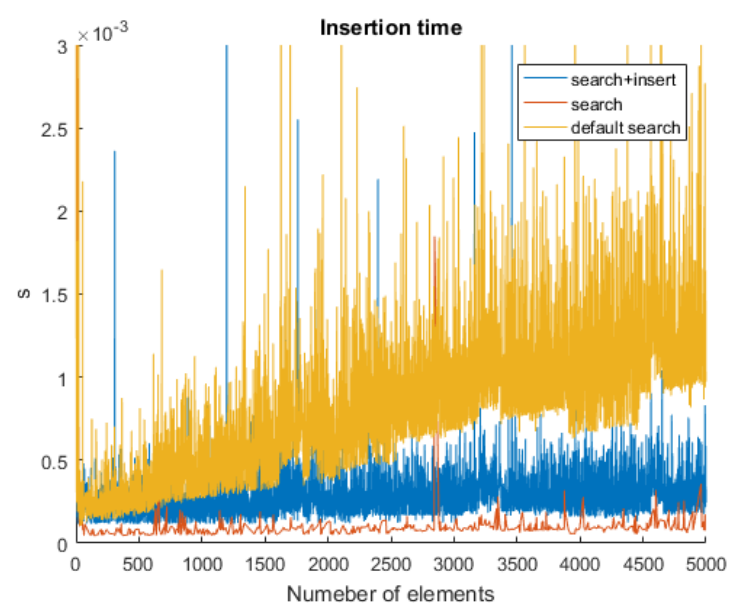

Figure 3. Insert-time and search-time using the hash-based set compared to the search-time considering a regular MATLAB matrix.

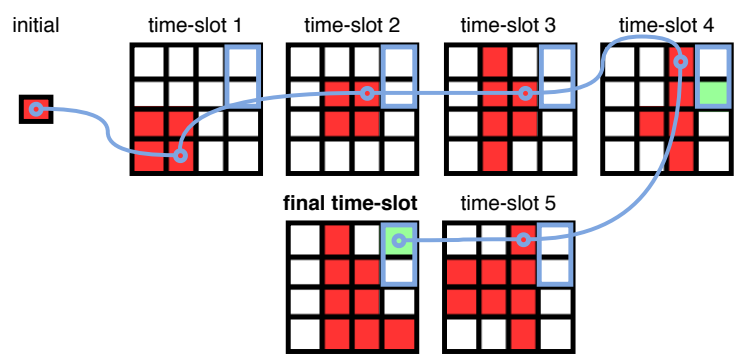

Figure 4. The No-Starvation MIMO Charging Problem aims at finding a path to any valid final charge-vector within the last time-slot.

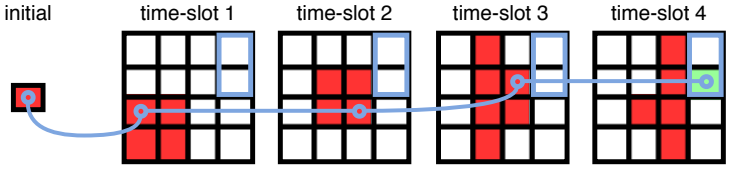

Figure 5. The Minimum-Time MIMO Charging Problem aims at finding the fastest path to any valid final charge-vector.

of the Minimum-Time MIMO Charging Problem is similar, as illustrated by Figure 5, but whatever the found valid last state, we stop populating the next sets and build the solution.

We created two methods for populating a charge-vector set from the previous one. The Exploration method generates a random $q_{t}$ charge-vector starting from a given $q_{t-1}$ and guarantees all constraints are respected. This method makes use of a closed formula with simple and inexpensive matrix calculations. Therefore, it is executed repeatedly to quickly fill the charge-vector space of the next time slot. The Exploitation method, in turn, tries finding an input-voltage vector from which the system reaches a given next state $q_{t}$ parting from a given previous state $q_{t-1}$. Thus, one may apply this method to fill holes in the charge-vector space or to obtain a well-behaved set. This method starts from a solution obtained using the Exploration method and iteratively approaches it to the targeted next state. It is more costly than the Exploration one, although it may require few iterations to converge, as shown in Figure 6.

We propose three algorithms based on the dynamic-programming approach, which are summarized as follows. (i) Simple Algorithm: consists of the literal application of the method and uses double-precision variables to store the memory structure, (ii) Pareto Algorithm: for each slot, stores a set of charge-vectors in such a way that no 


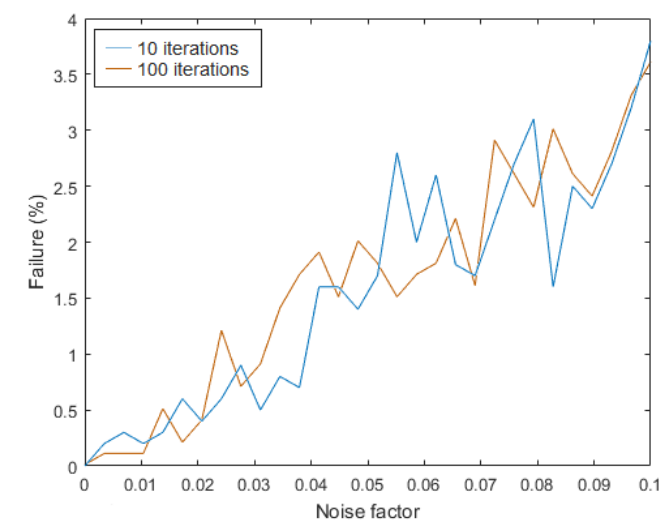

Figure 6. Percentage of failures for the exploitation method considering different numbers of iterations. The noise factor corresponds to the difference between the initial solution and the target.

vector is a multiple of another. The multiple is chosen so that the transferred power is maximized, and (iii) Fly-Weight Algorithm: consists of an adaptation of the Simple Algorithm designed to improve space-efficiency. Indeed, it requires only about $20 \%$ of the memory space used by the Simple Algorithm.

Finally, we propose three greedy algorithms. These are (i) Max-Sum-OfCurrents Algorithm: maximizes the sum of the charging currents of all batteries at each time-slot, (ii) Max-Sum Algorithm: maximizes the sum of the charges of all batteries at each time-slot, and (iii) Max-Min Algorithm: maximizes the life-time of the receiving device with weaker state-of-charge at each time-slot.

\section{Experimental Results}

In this dissertation, we evaluate the proposed algorithms via a large set of simulations. We run the simulations via MATLAB using the mathematical modeling described in Chapter 3 of the dissertation. We employ the random instance generator proposed in Section 6.1 to create instances with a guaranteed and known solution to both problems. For the NoStarvation MIMO Charging Problem, these simulations aim on estimating the success probability of each algorithm when solving a random instance. For the Minimum-Time MIMO Charging Problem, in turn, the main evaluated response-variable is the Normalized Charging Time, that is, the ratio between the charging-time of the found solution and the charging-time of the solution generated as a byproduct of the random instance. We employ different parameters to generate instances of different difficulties. In total, the simulations use 450 random instances.

Since the computational problems are proposed in this dissertation, there is no algorithm designed specifically for solving those. Thus, we choose the WPT runtime optimization algorithm MultiSpot to be the baseline, given its notoriety in recent literature. For justice sake, we abstract the parameter acquisition phase of the algorithm and provide the exact value of each necessary parameter. The other baseline is the Max Power Algorithm, which uses brute-force to find the voltage input for each transmitter that maximizes the transferred power, which is the most common objective-function in WPT literature.

Figure 7 shows some experimental results for the No-Starvation MIMO Charging 


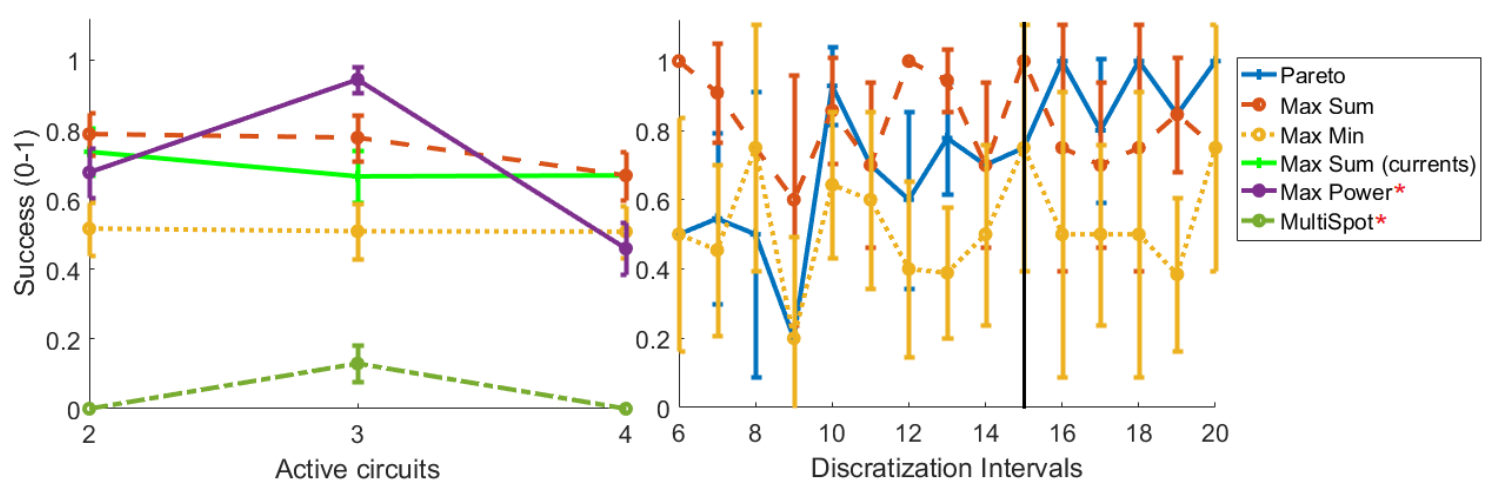

Figure 7. Comparison between the solutions generated by the proposed algorithms and the baseline $\left(^{*}\right)$ for instances of the No-Starvation MIMO Charging Problem. Over 15 charge discretization intervals (black vertical line), the dynamic-programming algorithms (represented here by Pareto) achieve better performance than greedy ones.

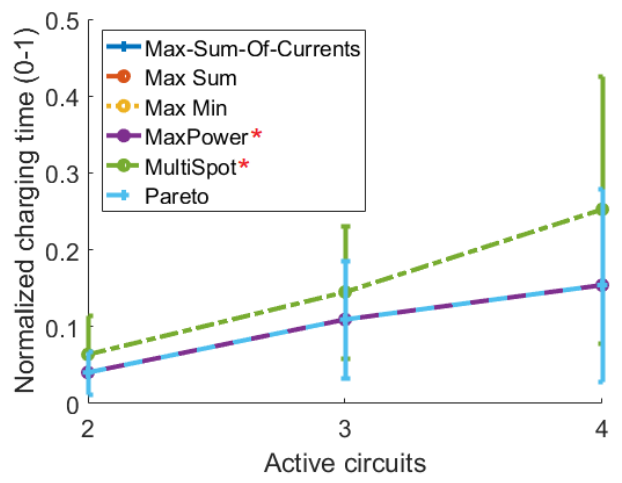

Figure 8. Comparison between the solutions generated by the proposed algorithms and the baseline (*) for instances of the Minimum-Time MIMO Charging Problem.

Problem. The most effective dynamic-programming algorithm is Pareto with at least 15 discretization intervals for the charge variables, whose success ratio varied between $89 \%$ and $97 \%$ for the tested scenarios. The most effective greedy algorithm is Max Sum, with success ratio between $74 \%$ and $92 \%$. The null hypothesis of these two algorithms reaching the same effectiveness can be rejected with $10 \%$ significance level using binomial test. Finally, the most effective baseline is Max Power, which surpassed 70\% in all scenarios. However, the null hypothesis of Max Power be equivalent to Max Sum is also rejected with $10 \%$ significance level against the hypothesis that Max Sum is superior.

Figure 8 shows some experimental results for the Minimum-Time MIMO Charging Problem. There is no evidence of improvements when comparing to the Max Power Algorithm, although MultiSpot is beaten by far by all other considered algorithms, since it does not include most constraints in their mathematical modeling.

\section{Publications}

1. de Sousa, Alexander; Vieira, Luiz ; Vieira, Marcos. Optimal transmission range and charging time for Qi-Compliant systems. IEEE Transactions on Power Electronics, pages 12765-12772, v.35, 2020. (Qualis A1) doi:10.1109/TPEL.2020.2996999 
2. de Sousa, Alexander; Vieira, Luiz ; Vieira, Marcos. Ubiquitous Wireless Power Transfer for Multiple Mobile Devices with no-starvation. IEEE Transactions on Mobile Computing, pages 1-12. (Qualis A1). In preparation.

3. de Sousa, Alexander; Vieira, Luiz ; Vieira, Marcos. Minimum-Time MIMO Charging Problem. IEEE Communications Magazine (Qualis A1). In preparation.

4. de Sousa, Alexander; Vieira, Luiz ; Vieira, Marcos. Communication Using Magnetic Induction. In: Fei Hu. (Org.). Magnetic Communications: From Theory to Practice. 1st ed., CRC Press, 2018, p.53-71, ISBN 9781498799782, .

\section{References}

Bekmezci, I., Sahingoz, O. K., and Temel, Ş. (2013). Flying ad-hoc networks (fanets): A survey. Ad Hoc Networks, 11(3):1254-1270.

Huang, L., Rieutort-Louis, W., Hu, Y., Sanz-Robinson, J., Wagner, S., Sturm, J. C., and Verma, N. (2012). Integrated all-silicon thin-film power electronics on flexible sheets for ubiquitous wireless charging stations based on solar-energy harvesting. In 2012 Symposium on VLSI Circuits (VLSIC), pages 198-199. IEEE.

Jadidian, J. and Katabi, D. (2014). Magnetic mimo: How to charge your phone in your pocket. In Proceedings of the 20th annual international conference on Mobile computing and networking, pages 495-506. ACM.

Lin, C., Guo, C., Dai, H., Wang, L., and Wu, G. (2019). Near optimal charging scheduling for 3-d wireless rechargeable sensor networks with energy constraints. In 2019 IEEE 39th International Conference on Distributed Computing Systems (ICDCS), pages 624-633. IEEE.

Madhja, A., Nikoletseas, S., Tsolovos, D., and Voudouris, A. A. (2018). Peer-to-peer energy-aware tree network formation. In Proceedings of the 16th ACM International Symposium on Mobility Management and Wireless Access, pages 1-8. ACM.

Nikoletseas, S., Raptis, T. P., and Raptopoulos, C. (2017). Wireless charging for weighted energy balance in populations of mobile peers. Ad Hoc Networks, 60:1-10.

Patil, A. (2020). Wireless charging market outlook - 2027. www.alliedmarketresearch.com/wireless-charging-market/ Accessed: 2020-05-16.

Shi, L., Kabelac, Z., Katabi, D., and Perreault, D. (2015). Wireless power hotspot that charges all of your devices. In Proceedings of the 21st Annual International Conference on Mobile Computing and Networking, pages 2-13. ACM.

Xie, L., Shi, Y., Hou, Y. T., and Sherali, H. D. (2012). Making sensor networks immortal: An energy-renewal approach with wireless power transfer. IEEE/ACM Transactions on networking, 20(6):1748-1761.

Zhao, C., Zhang, H., Chen, F., Chen, S., Wu, C., and Wang, T. (2020). Spatiotemporal charging scheduling in wireless rechargeable sensor networks. Computer Communications, 152:155-170. 